CLNS 04/1903

hep-ph/0412241

December 16, 2004

\title{
Two-Loop Relations for Heavy-Quark Parameters in the Shape-Function Scheme
}

\author{
Matthias Neubert \\ Institute for High-Energy Phenomenology \\ Newman Laboratory for Elementary-Particle Physics, Cornell University \\ Ithaca, NY 14853, U.S.A. \\ and \\ School of Natural Sciences, Institute for Advanced Study \\ Princeton, NJ 08540, U.S.A.
}

\begin{abstract}
Moments of the renormalized $B$-meson shape function provide a natural way to define short-distance, running heavy-quark parameters such as the $b$-quark mass and kinetic energy. These parameters are particularly well suited for studies of inclusive decay distributions. The definitions of $m_{b}$ and $\mu_{\pi}^{2}$ in this "shape-function scheme" are derived to two-loop order. Using previous determinations of heavy-quark parameters in other schemes, we find $m_{b}\left(\mu_{f}\right)=(4.63 \pm 0.08) \mathrm{GeV}$ and $\mu_{\pi}^{2}\left(\mu_{f}\right)=(0.15 \pm 0.07) \mathrm{GeV}^{2}$ at a reference scale $\mu_{f}=1.5 \mathrm{GeV}$.
\end{abstract}


Introduction. The past decade has seen a revolution in the precision with which fundamental physics can be probed using measurements of the decay properties of $b$ quarks. For instance, the element $\left|V_{c b}\right|$ of the quark mixing matrix is now known with a precision of $2 \%$, which is close to the accuracy of our knowledge of the Cabibbo angle [1]. Measurements of rare decay processes such as $B \rightarrow X_{s} \gamma$ impose stringent constraints on model building. The $b$-quark mass has been determined with an uncertainty of about $60 \mathrm{MeV}$ [1], which is much less than the QCD scale. To achieve such precision requires that heavy-quark parameters can be defined unambiguously using short-distance techniques. In particular, these definitions should not refer to the notion of on-shell quark states, which would introduce uncontrollable uncertainties ("renormalon ambiguities") that far exceed the experimental precision achieved at the $B$ factories.

The question of a short-distance definition of the $b$-quark mass has received much attention. While an ad hoc subtraction scheme such as $\overline{\mathrm{MS}}$ in principle defines the quark mass in an unambiguous way, such a definition is not appropriate for the discussion of $B$ decays, where the typical scales are often significantly below $m_{b}$. A more fruitful concept is that of a low-scale subtracted quark mass [2], which is based on the idea that non-perturbative contributions to the heavy-quark pole mass can be subtracted by making contact to some physical observable. The result is an expression $m_{b}\left(\mu_{f}\right)$, which differs from the pole mass by an amount proportional to a subtraction scale $\mu_{f}=$ few $\times \Lambda_{\mathrm{QCD}}$.

Several examples of such low-scale subtracted quark masses have been discussed in the literature. In the potential-subtraction scheme, long-distance contributions to the pole mass are subtracted by relating it to the static potential between two heavy quarks [3. Similarly, in the $\Upsilon(1 S)$ scheme the quark mass is related to the mass of the lowest-lying bottomonium resonance 4]. Both schemes are well suited to study heavy-quark systems in the non-relativistic regime, such as $b \bar{b}$ spectroscopy or heavy-quark production near threshold. A quark-mass definition more closely related to the non-perturbative physics probed in $B$-meson decays is provided by the kinetic scheme [2, 5, 6], in which the non-perturbative subtraction is accomplished with the help of heavy-quark sum rules [7. Because these sum rules constrain the properties of $B$-meson form factors in the "small-velocity limit", the kinetic scheme is well suited for heavy-quark expansions applied to $B$ decays into charm particles.

Inclusive $B$ decays into final states consisting of only light hadrons, such as $B \rightarrow X_{s} \gamma$ or $B \rightarrow X_{u} l \nu$, probe yet different aspects of non-perturbative physics. The bound-state effects relevant in these processes are encoded in $B$-meson shape functions defined in terms of the forward matrix elements of non-local string operators on the light cone [8, 9, 10]. A sensible definition of heavy-quark parameters for such processes should incorporate the bulk properties of the leading-order shape function $S(\omega)$. Schematically, inclusive decay spectra are given in terms of convolution integrals of the form

$$
\int d \omega f\left(m_{b}^{\text {pole }}+\omega\right) S(\omega) \approx f\left(m_{b}^{\text {pole }}+\langle\omega\rangle\right) \equiv f\left(m_{b}^{\mathrm{SF}}\right)
$$

where $f$ is some distribution, and in the last step we have defined the "shape-function mass" by adding the average value of $\omega$ to the pole mass. More precisely, the basis of the shape-function scheme proposed in [1] is to obtain short-distance definitions of all heavy-quark parameters 
that enter the description of inclusive decay rates via the moments

$$
M_{N}\left(\mu_{f}, \mu\right)=\int_{-\mu_{f}}^{\infty} d \omega \omega^{N} S(\omega, \mu)
$$

of the renormalized $B$-meson shape function $S(\omega, \mu)$, which has support over the range $-\infty<$ $\omega \leq m_{B}-m_{b}$. At tree level, the shape function is normalized to unity, its first moment vanishes (in the pole scheme), and higher moments are given in terms of local operator matrix elements in heavy-quark effective theory, e.g. $M_{2}^{\text {tree }}=-\lambda_{1} / 3$ [ㅁ, [10], where $\lambda_{1}$ is the kineticenergy parameter defined in the pole scheme [12. While it is well known that the $b$-quark pole mass suffers from a renormalon ambiguity, the same is true for other heavy-quark parameters such as $\lambda_{1}$ [13], even though this may not be obvious from low-order perturbative calculations 14. Beyond the tree approximation, the moments $M_{N}\left(\mu_{f}, \mu\right)$ depend on the renormalization scale $\mu$ and the lower cutoff $\mu_{f}$ applied to the integral in (2), and it is natural to use them to define a set of running heavy-quark parameters order by order in perturbation theory. In fact, for $\mu_{f} \gg \Lambda_{\mathrm{QCD}}$ these moments can be calculated using an operator product expansion, in which the scale $\mu_{f}$ plays the role of a hard Wilsonian cutoff. The moment integrals can then be matched onto a series of forward $B$-meson matrix elements of local operators. The Wilson coefficients in this matching can be obtained using perturbation theory with free quark and gluon states. In particular, if the matching is performed in the parton model with on-shell heavy-quark states $\left(v \cdot k=0\right.$, where $k=p_{b}-m_{b} v$ is the residual momentum, and $v$ denotes the $B$-meson velocity), then the local operator matrix elements are given by their tree-level expressions, and the matching becomes trivial once the moments (2) are computed to a given order in $\alpha_{s}$.

Two-loop relations in the shape-function scheme. The parton-model expression for the renormalized shape function was derived at one-loop order in [11, 15]. The result involves so-called star distributions [16, which are generalizations of plus distributions appropriate for test functions defined on an unbounded interval. This complication can be circumvented by introducing the function

$$
s(L, \mu)=\int_{-\mu_{f}}^{\infty} d \omega S_{\text {parton }}(\omega, \mu) ; \quad L=\ln \frac{\mu_{f}+n \cdot k}{\mu},
$$

whose dependence on the cutoff $\mu_{f}$ enters only through the logarithm $L$, while $\mu$ dependence also resides in the coupling $\alpha_{s}(\mu)$. Here $n$ is a light-like vector $\left(n^{2}=0, v \cdot n=1\right)$, which enters in the definition of the shape function as the matrix element of a non-local light-cone string operator 9]. The shape-function moments can now be obtained as

$$
M_{N}\left(\mu_{f}, \mu\right)=(-1)^{N}\left[\mu_{f}^{N} s(L, \mu)-N \int_{-n \cdot k}^{\mu_{f}} d \sigma \sigma^{N-1} s\left(\ln \frac{\sigma+n \cdot k}{\mu}, \mu\right)\right] .
$$

At the end of the calculation one must expand the result in powers of $n \cdot k$ and identify $n \cdot k \rightarrow 0$, $(n \cdot k)^{2} \rightarrow-\lambda_{1} / 3$, etc. [11].

The function $s(L, \mu)$ obeys an integro-differential renormalization-group equation, which can be derived starting from the evolution equation for the shape function obtained in [11, 17. 
We find

$$
\begin{aligned}
\frac{d}{d \ln \mu} s(L, \mu)= & 2\left[\Gamma_{\text {cusp }}\left(\alpha_{s}\right) L-\gamma\left(\alpha_{s}\right)\right] s(L, \mu) \\
& +2 \Gamma_{\text {cusp }}\left(\alpha_{s}\right) \int_{0}^{1} \frac{d z}{z}[s(L+\ln (1-z), \mu)-s(L, \mu)],
\end{aligned}
$$

where $\alpha_{s} \equiv \alpha_{s}(\mu)$, and $\Gamma_{\text {cusp }}, \gamma$ are anomalous dimensions. This equation can be solved order by order in perturbation theory with an ansatz of the form

$$
s(L, \mu)=1+\sum_{n=1}^{\infty}\left(\frac{\alpha_{s}(\mu)}{4 \pi}\right)^{n}\left(c_{0}^{(n)}+c_{1}^{(n)} L+\ldots+c_{2 n-1}^{(n)} L^{2 n-1}+c_{2 n}^{(n)} L^{2 n}\right) .
$$

The evolution equation (15) allows us to express all coefficients of logarithms in terms of the perturbative expansion coefficients of the anomalous dimensions and $\beta$ function, defined as

$$
\begin{aligned}
\Gamma_{\text {cusp }}\left(\alpha_{s}\right) & =\sum_{n=0}^{\infty} \Gamma_{n}\left(\frac{\alpha_{s}}{4 \pi}\right)^{n+1}, \quad \gamma\left(\alpha_{s}\right)=\sum_{n=0}^{\infty} \gamma_{n}\left(\frac{\alpha_{s}}{4 \pi}\right)^{n+1}, \\
\beta\left(\alpha_{s}\right) & =\frac{d \alpha_{s}}{d \ln \mu}=-2 \alpha_{s} \sum_{n=0}^{\infty} \beta_{n}\left(\frac{\alpha_{s}}{4 \pi}\right)^{n+1} .
\end{aligned}
$$

At two-loop order, we obtain

$$
\begin{aligned}
s(L, \mu)=1 & +\frac{\alpha_{s}(\mu)}{4 \pi}\left[c_{0}^{(1)}+2 \gamma_{0} L-\Gamma_{0} L^{2}\right] \\
& +\left(\frac{\alpha_{s}(\mu)}{4 \pi}\right)^{2}\left[c_{0}^{(2)}+\left(2 c_{0}^{(1)}\left(\gamma_{0}-\beta_{0}\right)+2 \gamma_{1}+\frac{2 \pi^{2}}{3} \Gamma_{0} \gamma_{0}+4 \zeta_{3} \Gamma_{0}^{2}\right) L\right. \\
& \left.+\left(2 \gamma_{0}\left(\gamma_{0}-\beta_{0}\right)-c_{0}^{(1)} \Gamma_{0}-\Gamma_{1}-\frac{\pi^{2}}{3} \Gamma_{0}^{2}\right) L^{2}+\left(\frac{2}{3} \beta_{0}-2 \gamma_{0}\right) \Gamma_{0} L^{3}+\frac{1}{2} \Gamma_{0}^{2} L^{4}\right] .
\end{aligned}
$$

The relevant expansion coefficients of the $\beta$ function and cusp anomalous dimension $\Gamma_{\text {cusp }}$ 18 are (in the $\overline{\mathrm{MS}}$ renormalization scheme)

$$
\beta_{0}=\frac{11}{3} C_{A}-\frac{2}{3} n_{f}, \quad \Gamma_{0}=4 C_{F}, \quad \Gamma_{1}=C_{F}\left[\left(\frac{268}{9}-\frac{4 \pi^{2}}{3}\right) C_{A}-\frac{40}{9} n_{f}\right] .
$$

The two-loop coefficient of the anomalous dimension $\gamma$ has been calculated in [19]. We have found some mistakes in the translation of the results for the two-loop graphs into the expression for the anomalous dimension. The corrected result is [17, 20]

$$
\gamma_{0}=-2 C_{F}, \quad \gamma_{1}=C_{F}\left[\left(\frac{74}{27}+\frac{\pi^{2}}{18}-18 \zeta_{3}+\kappa\right) C_{A}+\left(\frac{4}{27}+\frac{\pi^{2}}{9}\right) n_{f}\right],
$$

where $\kappa=0$ under the assumption that the two-loop diagrams themselves were evaluated correctly in [19]. However, there is reason to believe that there might be an additional error 
in that paper, giving rise to a non-zero value $\kappa=4 / 3$ [21], which we adopt in our numerical analysis. The expression for $\gamma_{1}$ should be checked with an independent calculation. Finally, the coefficient $c_{0}^{(1)}=\left(-\pi^{2} / 6\right) C_{F}$ was computed in [11, 15, while the coefficient $c_{0}^{(2)}$ is presently unknown. This coefficient does not enter the definitions of heavy-quark parameters at two-loop order in the shape-function scheme.

Using the explicit two-loop expression (8) for the function $s(L, \mu)$, it is straightforward to compute expressions for the shape-function moments (41) in the pole scheme. Closed expressions valid at one-loop order are given in [11. One finds that the first moment $M_{1}\left(\mu_{f}, \mu\right)$ no longer vanishes beyond the tree approximation but receives a perturbative correction proportional to $\mu_{f} \alpha_{s}(\mu)$. The key idea behind the shape-function scheme is to redefine the $b$-quark mass, $m_{b}^{\text {pole }} \equiv m_{b}\left(\mu_{f}, \mu\right)+\delta m$, in such a way that after the redefinition the first moment vanishes. Technically, this is implemented by introducing a residual mass term $\delta m$ for the heavy quark 22. All that changes in the expressions for the moments is that the residual momentum $k$ gets replaced by $k+\delta m v$, and hence $n \cdot k \rightarrow n \cdot k+\delta m$. We then choose $\delta m$ such that $M_{1}\left(\mu_{f}, \mu\right)=0$, thereby defining a mass renormalization scheme order by order in perturbation theory. Next, we define the kinetic-energy parameter $\mu_{\pi}^{2}\left(\mu_{f}, \mu\right)$ via the ratio $3 M_{2}\left(\mu_{f}, \mu\right) / M_{0}\left(\mu_{f}, \mu\right)$ in the new scheme. This quantity differs from the pole-scheme parameter $-\lambda_{1}$ by terms of order $\mu_{f}^{2} \alpha_{s}(\mu)$. We then solve for $m_{b}^{\text {pole }}$ and $\lambda_{1}$ in terms of the new parameters $m_{b}\left(\mu_{f}, \mu\right)$ and $\mu_{\pi}^{2}\left(\mu_{f}, \mu\right)$. To two-loop order, the results of these manipulations are

$$
\begin{aligned}
m_{b}^{\text {pole }}= & m_{b}\left(\mu_{f}, \mu\right)+\mu_{f} \frac{C_{F} \alpha_{s}(\mu)}{\pi}\left[1-2 \ln \frac{\mu_{f}}{\mu}+\frac{\alpha_{s}(\mu)}{\pi} k_{1}\left(\mu_{f}, \mu\right)\right] \\
& +\frac{\mu_{\pi}^{2}\left(\mu_{f}, \mu\right)}{3 \mu_{f}} \frac{C_{F} \alpha_{s}(\mu)}{\pi}\left[2 \ln \frac{\mu_{f}}{\mu}+\frac{\alpha_{s}(\mu)}{\pi} k_{2}\left(\mu_{f}, \mu\right)\right] \\
-\lambda_{1}= & \mu_{\pi}^{2}\left(\mu_{f}, \mu\right)\left[1+\frac{C_{F} \alpha_{s}(\mu)}{\pi}\left(-\frac{1}{2}-3 \ln \frac{\mu_{f}}{\mu}+\frac{\alpha_{s}(\mu)}{\pi} k_{3}\left(\mu_{f}, \mu\right)\right)\right] \\
& +\mu_{f}^{2} \frac{C_{F} \alpha_{s}(\mu)}{\pi}\left[3 \ln \frac{\mu_{f}}{\mu}+\frac{\alpha_{s}(\mu)}{\pi} k_{4}\left(\mu_{f}, \mu\right)\right]
\end{aligned}
$$

where the one-loop terms agree with [11, while the two-loop corrections are encoded in the coefficient functions

$$
\begin{aligned}
k_{1}\left(\mu_{f}, \mu\right)= & \frac{47}{36} \beta_{0}+\left(\frac{10}{9}-\frac{\pi^{2}}{12}-\frac{9}{4} \zeta_{3}+\frac{\kappa}{8}\right) C_{A}+\left(-8+\frac{\pi^{2}}{3}+4 \zeta_{3}\right) C_{F} \\
& +\left[-\frac{4}{3} \beta_{0}+\left(-\frac{2}{3}+\frac{\pi^{2}}{6}\right) C_{A}+\left(8-\frac{2 \pi^{2}}{3}\right) C_{F}\right] \ln \frac{\mu_{f}}{\mu}+\left(\frac{1}{2} \beta_{0}+2 C_{F}\right) \ln ^{2} \frac{\mu_{f}}{\mu} \\
k_{2}\left(\mu_{f}, \mu\right)= & -k_{1}\left(\mu_{f}, \mu\right)+\frac{7}{6} \beta_{0}+\left(\frac{1}{3}-\frac{\pi^{2}}{12}\right) C_{A}+\left(-5+\frac{\pi^{2}}{3}\right) C_{F}+\left(-\frac{1}{2} \beta_{0}-C_{F}\right) \ln \frac{\mu_{f}}{\mu} \\
k_{3}\left(\mu_{f}, \mu\right)= & \frac{1}{8} \beta_{0}+\left(1+\frac{\pi^{2}}{24}-\frac{27}{8} \zeta_{3}+\frac{3 \kappa}{16}\right) C_{A}+\left(-\frac{13}{4}-\frac{\pi^{2}}{6}+6 \zeta_{3}\right) C_{F}
\end{aligned}
$$




$$
\begin{aligned}
+ & {\left[-\beta_{0}+\left(-1+\frac{\pi^{2}}{4}\right) C_{A}+\left(\frac{27}{2}-\pi^{2}\right) C_{F}\right] \ln \frac{\mu_{f}}{\mu}+\left(\frac{3}{4} \beta_{0}+2 C_{F}\right) \ln ^{2} \frac{\mu_{f}}{\mu}, } \\
k_{4}\left(\mu_{f}, \mu\right)= & -k_{3}\left(\mu_{f}, \mu\right)-\frac{11}{24} \beta_{0}+\left(-\frac{1}{6}+\frac{\pi^{2}}{24}\right) C_{A}+\left(\frac{7}{2}-\frac{\pi^{2}}{6}\right) C_{F} \\
& +\left(\frac{1}{4} \beta_{0}-\frac{3}{2} C_{F}\right) \ln \frac{\mu_{f}}{\mu}-4 C_{F} \ln ^{2} \frac{\mu_{f}}{\mu} .
\end{aligned}
$$

Equations (111) and (12) are the main results of this work. They allow us to consistently implement the shape-function scheme with two-loop accuracy in perturbative calculations of heavy-quark processes. They also enable us to connect the shape-function scheme with any other short-distance scheme used to define heavy-quark parameters.

For practical applications, it is often inconvenient to use heavy-quark parameters defined as a function of two scales, and we will therefore adopt the special choice $\mu=\mu_{f}$ as our default, denoting $m_{b}\left(\mu_{f}\right) \equiv m_{b}\left(\mu_{f}, \mu_{f}\right)$ and $\mu_{\pi}^{2}\left(\mu_{f}\right) \equiv \mu_{\pi}^{2}\left(\mu_{f}, \mu_{f}\right)$. Then the relations (11) simplify, since all logarithms vanish. For $N_{c}=3$, we obtain

$$
\begin{aligned}
m_{b}^{\text {pole }}= & m_{b}\left(\mu_{f}\right)+\mu_{f} \frac{4 \alpha_{s}\left(\mu_{f}\right)}{3 \pi}\left[1+\frac{\alpha_{s}\left(\mu_{f}\right)}{\pi}\left(\frac{253}{36}+\frac{7 \pi^{2}}{36}-\frac{17}{12} \zeta_{3}+\frac{3 \kappa}{8}-\frac{47}{54} n_{f}\right)\right] \\
& +\frac{\mu_{\pi}^{2}\left(\mu_{f}\right)}{\mu_{f}}\left(\frac{\alpha_{s}\left(\mu_{f}\right)}{\pi}\right)^{2}\left(\frac{5}{81}+\frac{17}{27} \zeta_{3}-\frac{\kappa}{6}+\frac{10}{243} n_{f}\right), \\
-\lambda_{1}= & \mu_{\pi}^{2}\left(\mu_{f}\right)\left[1-\frac{2 \alpha_{s}\left(\mu_{f}\right)}{3 \pi}+\left(\frac{\alpha_{s}\left(\mu_{f}\right)}{\pi}\right)^{2}\left(\frac{1}{18}-\frac{7 \pi^{2}}{54}-\frac{17}{6} \zeta_{3}+\frac{3 \kappa}{4}-\frac{n_{f}}{9}\right)\right] \\
& +\mu_{f}^{2}\left(\frac{\alpha_{s}\left(\mu_{f}\right)}{\pi}\right)^{2}\left(-\frac{11}{9}+\frac{17}{6} \zeta_{3}-\frac{3 \kappa}{4}+\frac{14}{27} n_{f}\right) .
\end{aligned}
$$

Connections with other schemes. To the best of our knowledge, the only other scheme in which both $m_{b}$ and $\mu_{\pi}^{2}$ are defined in a consistent way (without renormalon ambiguities) is the kinetic scheme [2]. The relevant two-loop relations in that scheme are [5, 6]

$$
\begin{aligned}
m_{b}^{\text {pole }}= & m_{b}\left(\mu_{f}\right)+\frac{4}{3} \mu_{f} \frac{C_{F} \alpha_{s}\left(2 \mu_{f}\right)}{\pi}\left\{1+\frac{\alpha_{s}\left(2 \mu_{f}\right)}{\pi}\left[\frac{4}{3} \beta_{0}+\left(\frac{13}{12}-\frac{\pi^{2}}{6}\right) C_{A}\right]\right\} \\
& +\frac{\mu_{f}^{2}}{2 m_{b}\left(\mu_{f}\right)} \frac{C_{F} \alpha_{s}\left(2 \mu_{f}\right)}{\pi}\left\{1+\frac{\alpha_{s}\left(2 \mu_{f}\right)}{\pi}\left[\frac{13}{12} \beta_{0}+\left(\frac{13}{12}-\frac{\pi^{2}}{6}\right) C_{A}\right]\right\}, \\
-\lambda_{1}= & \mu_{\pi}^{2}\left(\mu_{f}\right)-\mu_{f}^{2} \frac{C_{F} \alpha_{s}\left(2 \mu_{f}\right)}{\pi}\left\{1+\frac{\alpha_{s}\left(2 \mu_{f}\right)}{\pi}\left[\frac{13}{12} \beta_{0}+\left(\frac{13}{12}-\frac{\pi^{2}}{6}\right) C_{A}\right]\right\} .
\end{aligned}
$$

The fact that the $b$-quark mass receives a $1 / m_{b}$ correction in the kinetic scheme leads to a mixing between different terms in the $1 / m_{b}$ expansion. No such mixing occurs in the shapefunction scheme. Other approaches such as the potential-subtraction or $\Upsilon(1 S)$ schemes only redefine the $b$-quark mass but ignore the problem of infrared ambiguities of other heavy-quark 
parameters. The two-loop relation between the potential-subtracted quark mass and the pole mass reads [3]

$$
m_{b}^{\text {pole }}=m_{b}\left(\mu_{f}\right)+\mu_{f} \frac{C_{F} \alpha_{s}\left(\mu_{f}\right)}{\pi}\left[1+\frac{\alpha_{s}\left(\mu_{f}\right)}{\pi}\left(\frac{11}{12} \beta_{0}-\frac{2}{3} C_{A}\right)\right] .
$$

At one-loop order, the potential-subtracted quark mass coincides with the mass defined (at the same scale $\mu_{f}$ ) in the shape-function scheme. The next-to-leading order relation between the pole mass and the $b$-quark mass defined in the $\Upsilon(1 S)$ scheme is [23]

$$
m_{b}=m_{b}^{\text {pole }}-\frac{\left[C_{F} \alpha_{s}(\mu)\right]^{2}}{8} m_{b}^{\text {pole }}\left\{1+\frac{\alpha_{s}(\mu)}{\pi}\left[\left(L+\frac{11}{6}\right) \beta_{0}-\frac{4}{3} C_{A}\right]\right\},
$$

where $L=\ln \left[\mu / C_{F} \alpha_{s}(\mu) m_{b}^{\text {pole }}\right]$. The peculiar counting of powers of $\alpha_{s}(\mu)$ in the $\Upsilon(1 S)$ scheme becomes more transparent if we rewrite this relation in the form

$$
m_{b}^{\text {pole }}=m_{b}+\mu_{f}(\mu) \frac{C_{F} \alpha_{s}(\mu)}{\pi}\left\{1+\frac{\alpha_{s}(\mu)}{\pi}\left[\left(\ln \frac{\pi \mu}{8 \mu_{f}(\mu)}+\frac{11}{6}\right) \beta_{0}-\frac{4}{3} C_{A}\right]\right\}
$$

where

$$
\mu_{f}(\mu)=\frac{\pi}{8} C_{F} \alpha_{s}(\mu) m_{b}^{\text {pole }}
$$

plays the role of the subtraction scale. Note that the $\Upsilon(1 S)$ mass is scale independent, contrary to the other low-scale subtracted quark masses considered above. On the other hand, the subtraction point $\mu_{f}(\mu)$ is itself a function of the renormalization scale $\mu$. Based on (16) one would suspect that a proper choice of $\mu$ is such that $L \approx 0$, which yields $\mu \approx 2 \mathrm{GeV}$. However, relation (18) suggests that the appropriate physical scale in the $\Upsilon(1 S)$ scheme may be lower, in accordance with arguments presented in 24$]$.

Numerical results. Estimates of the $b$-quark mass and kinetic energy in the shape-function scheme can be obtained using various sources of phenomenological information derived in other subtraction schemes. For this purpose we adopt the scale choice $\mu_{f}=\mu=1.5 \mathrm{GeV}$ in the shape-function scheme. The running of the heavy-quark parameters will be studied later. As mentioned above, we use $\kappa=4 / 3$.

The values of $m_{b}$ and $\mu_{\pi}^{2}$ defined in the kinetic scheme at a reference scale $\mu_{f}=1 \mathrm{GeV}$ have recently be determined from a global fit to experimental data on moments of various $B \rightarrow$ $X_{c} l \nu$ decay distributions [1], yielding $m_{b}=(4.611 \pm 0.068) \mathrm{GeV}$ and $\mu_{\pi}^{2}=(0.447 \pm 0.053) \mathrm{GeV}^{2}$ (with a mild anti-correlation of errors, $c=-0.4$ ), in good agreement with theoretical expectations 25. Using these results, we compute the pole scheme parameters from (14) and then solve for the parameters in the shape-function scheme using (13). We obtain

$$
m_{b}(1.5 \mathrm{GeV})=(4.63 \pm 0.07) \mathrm{GeV}, \quad \mu_{\pi}^{2}(1.5 \mathrm{GeV})=(0.15 \pm 0.06) \mathrm{GeV}^{2}
$$

where the errors reflect the uncertainties in the kinetic-scheme parameters only, and the correlation is the same as in the kinetic scheme. Note that we solve the relation for $\mu_{\pi}^{2}\left(\mu_{f}\right)$ in (13) exactly, rather than inverting it to $O\left(\alpha_{s}^{2}\right)$. (In the latter case the result for $m_{b}$ would 

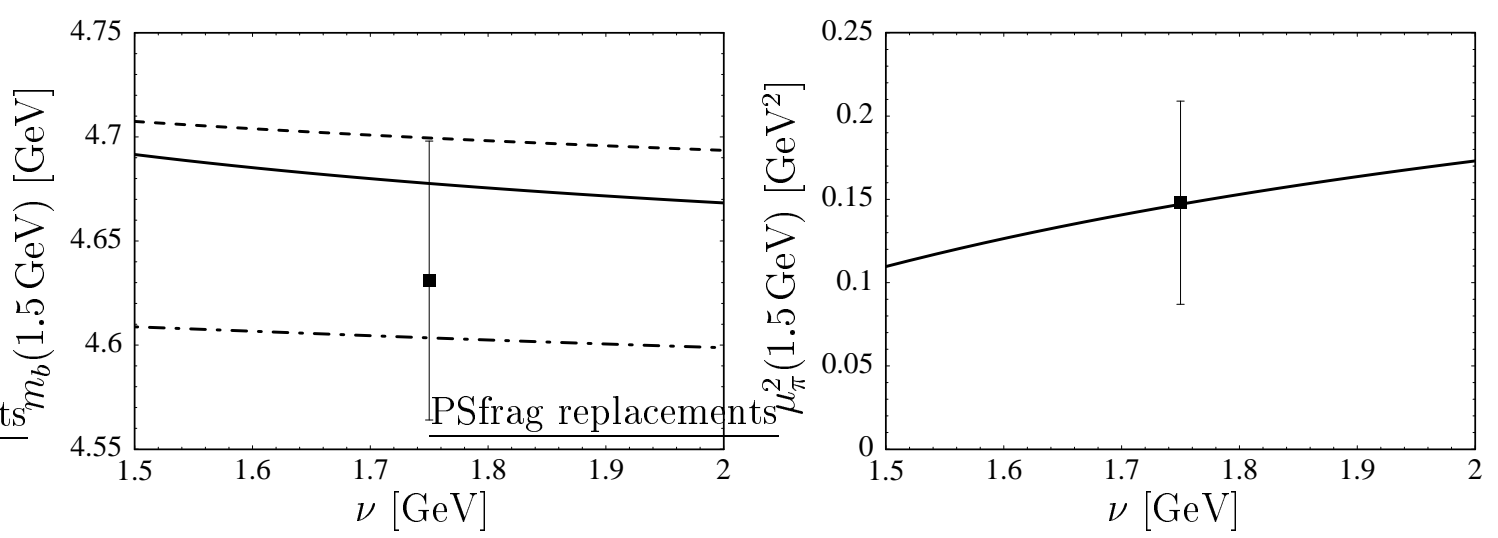

Figure 1: Dependence of the heavy-quark parameters in the shape-function scheme on the scale $\nu$ at which the coupling constant is evaluated. The lines refer to the translation of results obtained in the kinetic scheme (solid), the potential-subtraction scheme (dashed), and the $\Upsilon(1 S)$ scheme (dashed-dotted). Central values for the heavy-quark parameters in these schemes are used as input. The points show the values in (19) for comparison.

stay unchanged, whereas the value of $\mu_{\pi}^{2}$ would increase to $(0.18 \pm 0.06) \mathrm{GeV}^{2}$, which is inside the error range.) For reference, the corresponding results obtained at one-loop order are $m_{b}(1.5 \mathrm{GeV})=(4.57 \pm 0.07) \mathrm{GeV}$ and $\mu_{\pi}^{2}(1.5 \mathrm{GeV})=(0.34 \pm 0.06) \mathrm{GeV}^{2}$. The significant impact of two-loop corrections in the case of $\mu_{\pi}^{2}$ is due to the terms proportional to $\mu_{f}^{2}$ in the relation for $\lambda_{1}$ in (13), which arise first at $O\left(\alpha_{s}^{2}\right)$.

The potential-subtracted mass at the scale $\mu_{f}=2 \mathrm{GeV}$ has been determined from an analysis of the $b \bar{b}$ production cross section and the mass of the $\Upsilon(1 S)$ resonance to be $m_{b}=$ $(4.59 \pm 0.08) \mathrm{GeV}$ [24]. Using relations (13) and (15), as well as the value of $\mu_{\pi}^{2}$ given in (19), we may use this information to obtain $m_{b}(1.5 \mathrm{GeV})=(4.66 \pm 0.08) \mathrm{GeV}$ for the $b$ quark mass in the shape-function scheme, in excellent agreement with (19). We may also use the value $m_{b}=(4.68 \pm 0.03) \mathrm{GeV}$ obtained in the $\Upsilon(1 S)$ scheme from a fit to moments of $B \rightarrow X_{c} l \nu$ and $B \rightarrow X_{s} \gamma$ decay distributions [26], even though we do not trust the very small error assigned in this analysis. Taking $\mu=1.5 \mathrm{GeV}$ for the scale in (16), we find $m_{b}(1.5 \mathrm{GeV})=(4.61 \pm 0.03) \mathrm{GeV}$ for the $b$-quark mass in the shape-function scheme, which is again consistent with (19).

The numbers presented above are affected by additional theoretical uncertainties inherent in the translation from one scheme to another. A way of assessing these uncertainties is to evaluate the coupling constant $\alpha_{s}(\nu)$ in the various relations at a common scale $\nu$ different from the individual "natural" scales. This can be done using

$$
\alpha_{s}(\mu)=\alpha_{s}(\nu)\left[1+\frac{\beta_{0}}{2} \frac{\alpha_{s}(\nu)}{\pi} \ln \frac{\nu}{\mu}+\ldots\right] .
$$

While the results are formally independent of the choice of $\nu$, the variations due to truncation effects may serve as an indicator of the impact of higher-order effects. Varying $\nu$ between 1.5 and $2 \mathrm{GeV}$ so as to cover the range of "natural" scales in the various schemes yields the results 
shown in Figure 1. The perturbative uncertainties indicated by this analysis are somewhat smaller than the parameter uncertainties shown in (19). Combining the two sources of errors, we quote our final results as

$$
m_{b}(1.5 \mathrm{GeV})=(4.63 \pm 0.08) \mathrm{GeV}, \quad \mu_{\pi}^{2}(1.5 \mathrm{GeV})=(0.15 \pm 0.07) \mathrm{GeV}^{2}
$$

Ultimately, the shape-function parameters should be determined directly from experimental data, without a detour through another subtraction scheme. Moments of the photon energy spectrum in $B \rightarrow X_{s} \gamma$ decay are ideally suited for this purpose and will allow an extraction of $m_{b}$ with far better precision [27].

Given values of the heavy-quark parameters $m_{b}$ and $\mu_{\pi}^{2}$ defined in the shape-function scheme at some reference scale $\mu_{f}$, it is straightforward to solve the relations (13) to find the corresponding running parameters at a different scale. There is nothing unphysical about this scale dependence, which indeed is an essential feature of our approach. For instance, we obtain $m_{b}(1.1 \mathrm{GeV})=(4.64 \pm 0.08) \mathrm{GeV}$ and $\mu_{\pi}^{2}(1.1 \mathrm{GeV})=(0.18 \pm 0.07) \mathrm{GeV}^{2}$ at a lower scale, which is appropriate for applications the $B \rightarrow X_{s} \gamma$ photon spectrum [17, 27], and $m_{b}(2 \mathrm{GeV})=(4.60 \pm 0.08) \mathrm{GeV}$ and $\mu_{\pi}^{2}(2 \mathrm{GeV})=(0.11 \pm 0.07) \mathrm{GeV}^{2}$ at a higher scale, which could be used to analyse moments of $B \rightarrow X_{c} l \nu$ decay distributions. More important evolution effects are encountered when the scales $\mu_{f}$ and $\mu$ are taken differently, in which case the relations (11) must be used to compute the effects of scale changes. For example, with $\mu_{f}=1.1 \mathrm{GeV}$ and $\mu=1.5 \mathrm{GeV}$ we get $m_{b}(1.1 \mathrm{GeV}, 1.5 \mathrm{GeV})=(4.58 \pm 0.08) \mathrm{GeV}$ and $\mu_{\pi}^{2}(1.1 \mathrm{GeV}, 1.5 \mathrm{GeV})=(0.36 \pm 0.06) \mathrm{GeV}^{2}$.

Conclusions. In summary, we have derived the two-loop relations for the $b$-quark mass $m_{b}$ and kinetic-energy parameter $\mu_{\pi}^{2}$ in the shape-function scheme introduced in [11]. These relations are important for performing high-precision studies of $B$-decay observables. We have determined values for $m_{b}\left(\mu_{f}\right)$ and $\mu_{\pi}^{2}\left(\mu_{f}\right)$ at a reference scale $\mu_{f}=1.5 \mathrm{GeV}$ by using as input the corresponding values of heavy-quark parameters in other subtraction schemes. In the future, the shape-function parameters should be determined directly from a fit to moments of the $B \rightarrow X_{s} \gamma$ photon spectrum.

Acknowledgments. We are indebted to Einan Gardi for many useful discussions, which have been instrumental in finding the (hopefully correct) expressions for the two-loop anomalous dimension in (10). We are also grateful to Kolya Uraltsev for discussions on the kinetic scheme. This research was supported by the National Science Foundation under Grant PHY0355005, and by the Department of Energy under Grant DE-FG02-90ER40542.

Note added. After this work was submitted for publication, an Erratum to [19] appeared, in which the authors confirm the result (10) with $\kappa=4 / 3$.

\section{References}

[1] B. Aubert et al. [BaBar Collaboration], Phys. Rev. Lett. 93, 011803 (2004) hep-ex/0404017. 
[2] I. I. Y. Bigi, M. A. Shifman, N. Uraltsev and A. I. Vainshtein, Phys. Rev. D 56, 4017 (1997) hep-ph/9704245.

[3] M. Beneke, Phys. Lett. B 434, 115 (1998) hep-ph/9804241.

[4] A. H. Hoang, Z. Ligeti and A. V. Manohar, Phys. Rev. D 59, 074017 (1999) hep-ph/9811239.

[5] A. Czarnecki, K. Melnikov and N. Uraltsev, Phys. Rev. D 57, 1769 (1998) hep-ph/9706311.

[6] A. Czarnecki, K. Melnikov and N. Uraltsev, Phys. Rev. Lett. 80, 3189 (1998) hep-ph/9708372.

[7] I. I. Y. Bigi, M. A. Shifman, N. G. Uraltsev and A. I. Vainshtein, Phys. Rev. D 52, 196 (1995) hep-ph/9405410.

[8] M. Neubert, Phys. Rev. D 49, 3392 (1994) hep-ph/9311325.

[9] M. Neubert, Phys. Rev. D 49, 4623 (1994) hep-ph/9312311.

[10] I. I. Y. Bigi, M. A. Shifman, N. G. Uraltsev and A. I. Vainshtein, Int. J. Mod. Phys. A 9, 2467 (1994) hep-ph/9312359.

[11] S. W. Bosch, B. O. Lange, M. Neubert and G. Paz, Nucl. Phys. B 699, 335 (2004) hep-ph/0402094.

[12] A. F. Falk and M. Neubert, Phys. Rev. D 47, 2965 (1993) hep-ph/9209268.

[13] M. Neubert, Phys. Lett. B 393, 110 (1997) hep-ph/9610471.

[14] G. Martinelli, M. Neubert and C. T. Sachrajda, Nucl. Phys. B 461, 238 (1996) hep-ph/9504217.

[15] C. W. Bauer and A. V. Manohar, Phys. Rev. D 70, 034024 (2004) hep-ph/0312109.

[16] F. De Fazio and M. Neubert, JHEP 9906, 017 (1999) hep-ph/9905351.

[17] M. Neubert, hep-ph/0408179, Eur. Phys. J. C (in press).

[18] G. P. Korchemsky and A. V. Radyushkin, Nucl. Phys. B 283, 342 (1987); I. A. Korchemskaya and G. P. Korchemsky, Phys. Lett. B 287, 169 (1992).

[19] G. P. Korchemsky and G. Marchesini, Nucl. Phys. B 406, 225 (1993) hep-ph/9210281.

[20] We are grateful to G. Korchemsky for confirming these results (private communication).

[21] E. Gardi reports that he has confirmed (10) with $\kappa=4 / 3$ from an independent calculation of the anomalous dimension $\gamma$ (private communication). 
[22] A. F. Falk, M. Neubert and M. E. Luke, Nucl. Phys. B 388, 363 (1992) hep-ph/9204229.

[23] A. H. Hoang and T. Teubner, Phys. Rev. D 60, 114027 (1999) hep-ph/9904468.

[24] M. Beneke and A. Signer, Phys. Lett. B 471, 233 (1999) hep-ph/9906475.

[25] P. Gambino and N. Uraltsev, Eur. Phys. J. C 34, 181 (2004) hep-ph/0401063.

[26] C. W. Bauer, Z. Ligeti, M. Luke, A. V. Manohar and M. Trott, Phys. Rev. D 70, 094017 (2004) hep-ph/0408002.

[27] M. Neubert, Model-Independent Predictions for Moments of the $\bar{B} \rightarrow X_{s} \gamma$ Photon Spectrum, preprint CLNS 04/1904, in preparation. 